https://helda.helsinki.fi

\title{
Local adventitial anti-angiogenic gene therapy reduces growth of vasa-vasorum and in-stent restenosis in WHHL rabbits
}

\section{Hytonen, Jarkko P.}

2018-08

Hytonen , J P , Taavitsainen , J , Laitinen , J T T, Partanen , A , Alitalo , K, Leppänen , O \& Ylä-Herttuala , S 2018 , ' Local adventitial anti-angiogenic gene therapy reduces growth of vasa-vasorum and in-stent restenosis in WHHL rabbits ' , Journal of Molecular and Cellular Cardiology , vol. 121 , pp. 145-154 . https://doi.org/10.1016/j.yjmcc.2018.07.007

http://hdl.handle.net/10138/305124

https://doi.org/10.1016/j.yjmcc.2018.07.007

publishedVersion

Downloaded from Helda, University of Helsinki institutional repository.

This is an electronic reprint of the original article.

This reprint may differ from the original in pagination and typographic detail.

Please cite the original version. 
Original article

\title{
Local adventitial anti-angiogenic gene therapy reduces growth of vasa- vasorum and in-stent restenosis in WHHL rabbits
}

\author{
Jarkko P. Hytönen $^{\mathrm{a}, 1}$, Jouni Taavitsainen ${ }^{\mathrm{a}, 1}$, Johannes T.T. Laitinen ${ }^{\mathrm{a}}$, Anna Partanen ${ }^{\mathrm{a}}$, \\ Kari Alitalo $^{\mathrm{b}}$, Olli Leppänen ${ }^{\mathrm{c}}$, Seppo Ylä-Herttuala ${ }^{\mathrm{a}, \mathrm{d}, \mathrm{e}, *}$ \\ a A.I.Virtanen Institute for Molecular Sciences, University of Eastern Finland, Kuopio, Finland \\ b Translational Cancer Biology Program, Wihuri Research Institute, Biomedicum Helsinki, University of Helsinki, Helsinki, Finland \\ ${ }^{c}$ Centre for R\&D, Uppsala University/County Council of Gävleborg, Gävle, Sweden \\ ${ }^{\mathrm{d}}$ Heart Center, Kuopio University Hospital, Kuopio, Finland \\ e Gene Therapy Unit, Kuopio University Hospital, Kuopio, Finland
}

\section{A R T I C L E I N F O}

\section{Keywords:}

In-stent restenosis

Gene therapy

Plaque angiogenesis

Re-endothelialization

\begin{abstract}
A B S T R A C T
Background: Antiproliferative drugs in drug eluting stents (DES) are associated with complications due to impaired re-endothelialization. Additionally, adventitial neovascularization has been suggested to contribute to instent restenosis (ISR). Since Vascular Endothelial Growth Factors (VEGFs) are the key mediators of angiogenesis, we investigated feasibility and efficacy of local gene therapy for ISR utilizing soluble decoy VEGF receptors to reduce biological activity of adventitial VEGFs.

Method: Sixty-nine adult WHHL rabbit aortas were subjected to endothelial denudation. Six weeks later catheter-mediated local intramural infusion of $1.5 \times 10 \mathrm{e} 10 \mathrm{pfu}$ adenoviruses encoding soluble VEGF Receptor-1 (sVEGFR1), sVEGFR2, sVEGFR3 or control LacZ and bare metal stent implantation were performed in the same aortic segment. Marker protein expression was assessed at $6 \mathrm{~d}$ in LacZ cohort. Immunohistochemistry, morphometrical analyses and angiography were performed at d14, d42 and d90.

Results: Transgene expression was localized to adventitia. All decoy receptors reduced the size of vasa-vasorum at 14d, AdsVEGFR2 animals also had reduced density of adventitial vasa-vasorum, whereas AdsVEGFR3 increased the density of vasa-vasorum. At d42, AdsVEGFR1 and AdsVEGFR2 reduced ISR (15.7 \pm 6.9\% stenosis, $\mathrm{P}<0.01$ and $16.5 \pm 2.7 \%, \mathrm{P}<0.05$, respectively) vs. controls $(28.3 \pm 7.6 \%)$. Moreover, AdsVEGFR-3 treatment led to a non-significant trend in the reduction of adventitial lymphatics at all time points and these animals had significantly more advanced neointimal atherosclerosis at $14 \mathrm{~d}$ and $42 \mathrm{~d}$ vs. control animals. Conclusions: Targeting adventitial neovascularization using sVEGFR1 and sVEGFR2 is a novel strategy to reduce ISR. The therapeutic effects dissipate at late follow up following short expression profile of adenoviral vectors. However, inhibition of VEGFR3 signaling accelerates neoatherosclerosis.
\end{abstract}

\section{Introduction}

Percutaneous revascularization techniques have revolutionized the treatment of patients suffering from coronary artery disease [1,2]. The introduction of stents prevented elastic recoil and negative remodeling of coronary arteries after angioplasty [3], a key limitation of catheter based treatments until that time. Atherosclerotic disease and mechanical injury caused by the stent itself, however, commonly leads to a new narrowing [4]. This in-stent restenosis (ISR) has been reduced by the discovery of drug eluting stents (DES) [5]. The strong cytotoxic drugs used in DES commonly inhibit ISR, but also delay recovery of the protective endothelial layer leading to prolonged risk of stent thrombosis (ST) and reliance on antithrombotic medication [6].

Plaque angiogenesis has been suggested as one of the major factors in the progression of atherosclerotic lesions [7] and may be driven by hypoxia and energy depletion in macrophage-rich lesion core $[8,9]$. Intimal and medial cells activate angiogenesis by secretion of several growth factors including Vascular Endothelial Growth Factor A (VEGF-

\footnotetext{
Abbreviations: BMS, bare metal stent; DES, drug eluting stent; ISR, in-stent restenosis; VEGF, vascular endothelial growth factor; VEGFR, vascular endothelial growth factor receptor; WHHL, Watanabe Heritable Hyperlipidemic

* Corresponding author at: Department of Molecular Medicine, A.I.Virtanen- Institute, University of Eastern Finland, P.O.Box 1627, FIN-70210 Kuopio, Finland.

E-mail address: Seppo.Ylaherttuala@uef.fi (S. Ylä-Herttuala).

${ }^{1}$ Authors with equal contribution
} 

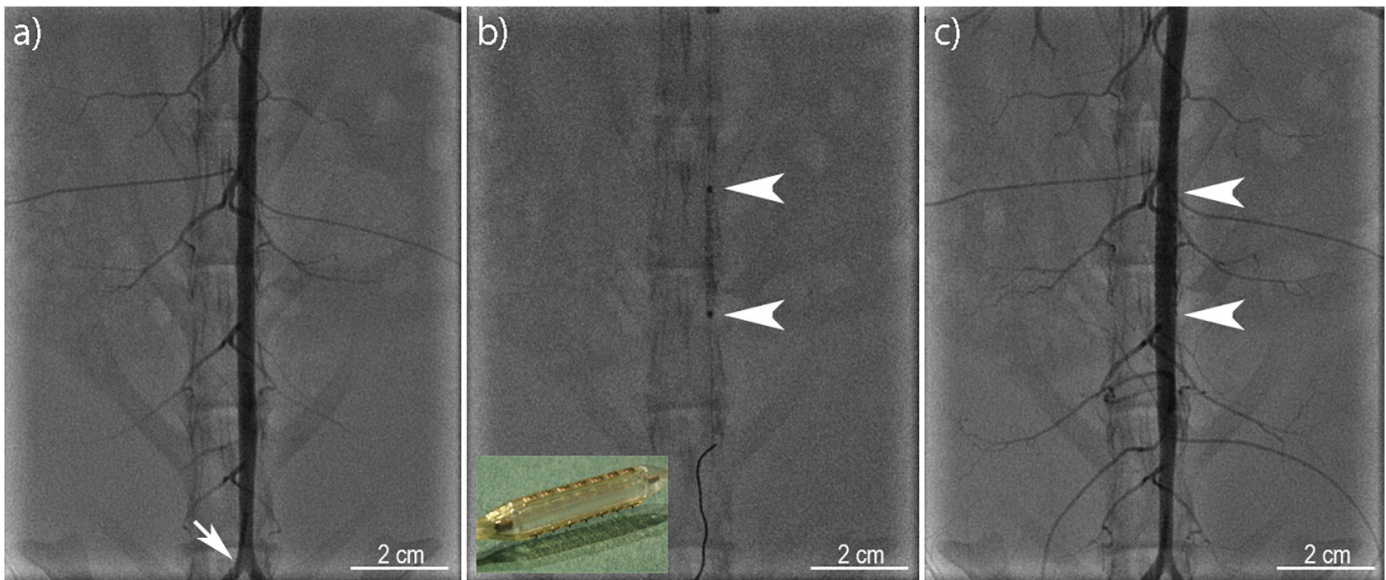

Fig. 1. Stent implantation.

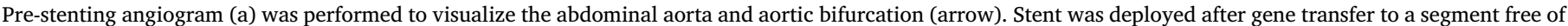

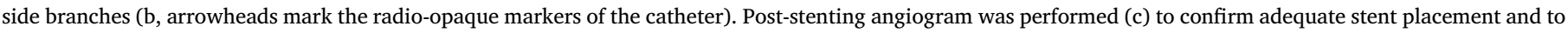
exclude dissection or thromboembolic complications (stent marked with arrowheads). Insert in b shows an inflated drug delivery balloon.

A) $[10,11]$. This is aggravated by growth factor and cytokine secretion from inflammatory cells, also found in abundance in atherosclerotic lesions. VEGF-A signaling is mediated through VEGFR1 and VEGFR2 with VEGFR1 acting as a decoy receptor as well as transducing weak signals for endothelial growth. VEGFR2 is a key mediator of endothelial cell survival and angiogenesis [12]. VEGF-A signaling leads to the growth of new vasa vasorum from the adventitial layer of the artery. Neoangiogenic capillaries are often poorly organized and leaky, which can lead to small hemorrhages in the growing lesion and to rupture prone, vulnerable plaques [9-11]. While abundant vasa vasorum have been linked to the growth of atherosclerotic lesions [11], and although descriptive studies have reported a correlation between the vasa vasorum density and the percent luminal stenosis after balloon injury [13], the role of plaque angiogenesis in the formation of ISR has remained elusive.

Similarly the role of lymphangiogenesis and VEGFR3 signaling, the main driver for the growth of lymphatic vessels, is unclear in the ISR formation. In vivo VEGFR3 activation by VEGF-C and VEGF-D triggers the growth of lymphatic vessels. In experimental setting reduced activity of VEGF-C has been shown to elevate cholesterol levels and accelerate atherogenesis [14]. However, the effects of lymphangiogenesis and lymphatic structures on ISR are unknown.

We studied the effects of anti-angiogenic therapy on the development of ISR in a clinically relevant triple injury model [15]. Adenoviruses encoding soluble decoy receptors (sVEGFR) 1, 2 and 3 or control LacZ were infused intramurally into the vascular wall at the time of stenting. Analyses of the control animals at $6 \mathrm{~d}$ demonstrated the marker protein expression in the outer layers of media and in the adventitia. sVEGFRs expressed by cells in the abluminal vessel layers act as secreted decoy receptors and bind to VEGFs, thus limiting their biological activity and reducing angiogenesis and lymphangiogenesis [16]. We hypothesized that adventitial sVEGFR overexpression would lead to attenuated angiogenesis or vasa vasorum growth and ultimately have an effect on in-stent restenosis.

\section{Methods}

\subsection{Adenoviruses}

Replication-deficient E1-partially E3-deleted clinical GMP-grade adenoviruses encoding nuclear-targeted $\beta$-galactosidase (LacZ) or soluble VEGF receptors 1,2 and 3 were produced and tested to be free of contaminants [17]. The same adenoviral constructs have been studied previously and shown to produce transgene products and be biologically active in-vivo [18].

\subsection{Animal model}

Animal experiments were approved by the University of Eastern Finland Research Ethics Committee and conforms to the National Institutes of Health guidelines. Adult (six month old) Watanable Heritable Hyperlipidemic (WHHL) rabbits $(n=69)$, with mutated LDL receptor gene resulting in high circulating cholesterol levels [15], were randomized into study groups. The aortas of the animals were denuded of endothelium via the right femoral artery with three passes of inflated 3F embolectomy catheter (Fogarty arterial embolectomy catheter, Edwards Lifesciences) under ketamine $(15 \mathrm{mg} / \mathrm{kg}$, Ketalar, Pfizer) and medetomidine $(0.3 \mathrm{mg} / \mathrm{kg}$, Domitor, Orion Pharma) anesthesia [15]. Denudation was performed between the level of the renal arteries and aortic bifurcation. Six weeks after the denudation the rabbits were anesthetized again and a $6 \mathrm{~F}$ introducer sheath (Avanti + , Cordis) was placed into the right carotid artery exposed through a mid-line incision. A guiding catheter (Vista Brite Tip, Cordis) and a guide wire (Stabilizer, Cordis) were advanced to the descending aorta under fluoroscopic guidance (GE Innova 3100IQ, GE Healthcare). Angiography was performed, the guiding catheter removed and Infiltrator ${ }^{\circledR}$ (IVT, Boston Scientific) drug delivery catheter advanced to the aorta. A segment in the distal parts of the aorta free of side branches was selected. The drug delivery catheter was moved to the selected segment and inflated according to manufacturer's instructions. Two milliliters of diluted virus solution with $1.5 \times 10 \mathrm{e} 10 \mathrm{pfu}$ of either AdLacZ, AdsVEGFR1, AdsVEGFR2 or AdsVEGFR3 was infused through the 0.01" high injection needles of the catheter over two minutes. The catheter was deflated and replaced with a straight guiding catheter and a bare metal stent $(3.5 \times 18 \mathrm{~mm}$, Multi-Link Zeta, Abbott Vascular) was deployed. The stent was positioned over the gene transfer site and inflated to accommodate the vessel in an approximately 1.1:1 ratio. Angiography was repeated to confirm appropriate stent placement (Fig. 1). Angiography was repeated before sacrifice 14,42 or 90 days after the stent implantation. An additional cohort receiving AdLacZ was sacrificed six days after the gene transfer to document gene transfer efficacy and assess the distribution of transgene expression. At the end of the follow up, the animals were sacrificed with a large i.v. bolus of saturated $\mathrm{MgSO}_{4}$ under general anesthesia and the vasculature was perfused with PBS. The stented segment of the aorta was fixed with paraformaldehyde and mounted in methacrylate and processed further for histopathological analyses [15]. Tissue samples from the heart, lung, liver, spleen, kidney and skeletal muscle were harvested for general histology and 

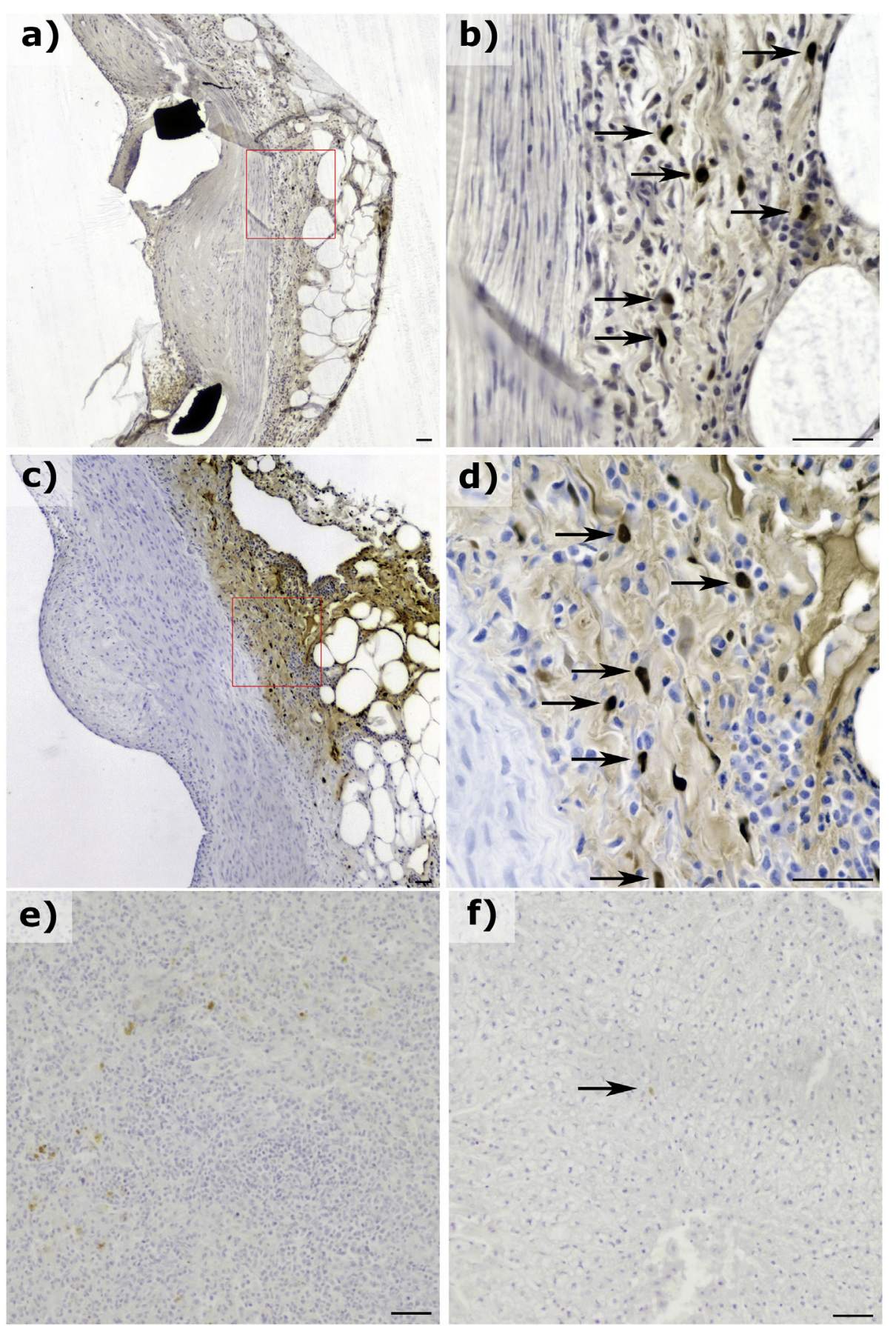

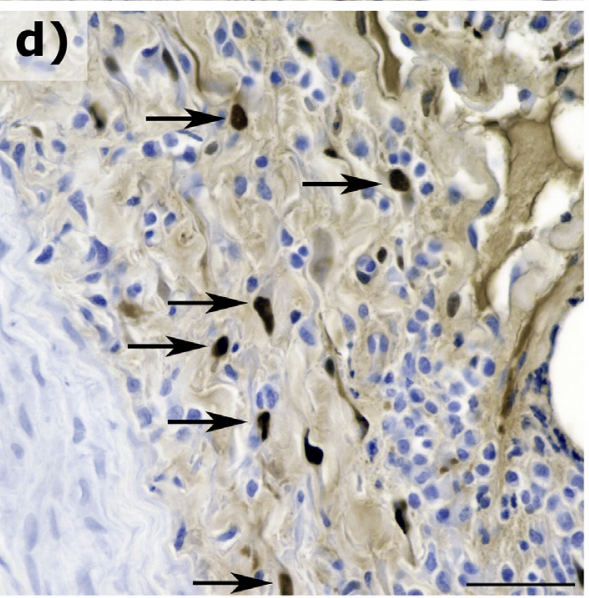

f)

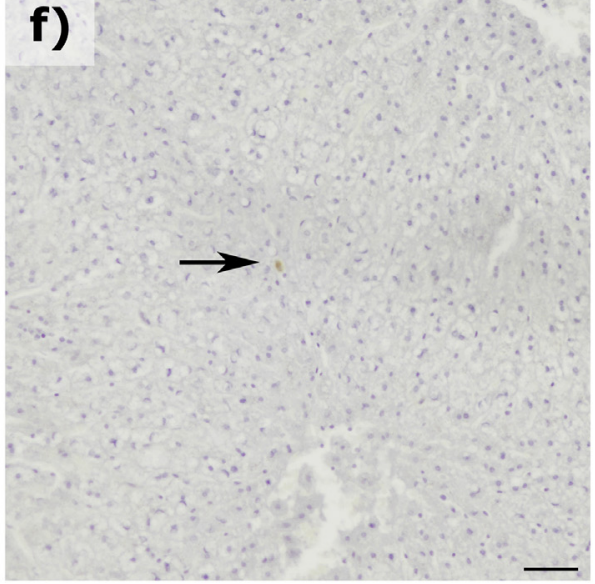

Fig. 2. Marker gene expression.

Immunohistology from AdLacZ treated stent six days after gene transfer and stenting with $\beta$-galactosidase staining. Positive cells are seen in the adventitia of the stented segments ( $a$ and $b$, higher magnification from indicated area shown in b) as well as arterial segments at the edges of the stent (c and d, higher magnification in d) (dark brown staining). Positive staining is also seen in the spleen (e) and liver (f) six days after treatment. Magnification $40 \times$ for a and c, $100 \times$ for e and f, $200 \times$ for $b$ and $d$. Scale bars $100 \mu \mathrm{m}$. Arrows in b, d, and $\mathrm{f}$ indicate positive cells. (For interpretation of the references to colour in this figure legend, the reader is referred to the web version of this article.) biodistribution analyses and were embedded into paraffin blocks after tissue fixation and dehydration. Segments of aorta from the edges of the stent were processed through paraffin histology and stained for $\beta$-galactosidase from d6 animals.

\subsection{Histology}

Stents were segmentally cut into $\leq 7 \mu \mathrm{m}$ serial sections and stained with hematoxylin and eosin as well as Van Gieson stain for elastin [15]. Immunohistological stains were performed with antibodies against endothelial cells (CD31, DAKO), lymphatic endothelium (LYVE-1, DAKO), rabbit macrophages (RAM-11, DAKO), proliferating cells (Ki67, DAKO), $\beta$-galactosidase (Promega) and smooth muscle cell actin (HHF-35, Enzo). Following treatment with the primary antibody samples were incubated with a biotinylated secondary antibody followed by avidin-biotin complex. Visualization was achieved with the DAB peroxidase system (Vector Laboratories). All immunohistological stainings were performed with hematoxylin counterstaining. Analyses were performed from all stents from a single layer along the stent. Images were acquired with Olympus AX70 microscope (Olympus Optical) and quantitative analyses performed with AnalySIS software (Soft Imaging System).

Adventitial vessels were quantified from CD31 stained sections. CD31 positive vessels were counted and their cross-sectional area measured from the adventitia $[15,19]$. The number of vessels is expressed as number of vessels per $\mathrm{mm}^{2}$ of tissue. Similar measurements were carried out for lymphatic vessels from LYVE-1 stained samples [14]. Endothelialization was determined from CD31 stained sections as the percentage of luminal circumference covered by CD31 positive cells. 

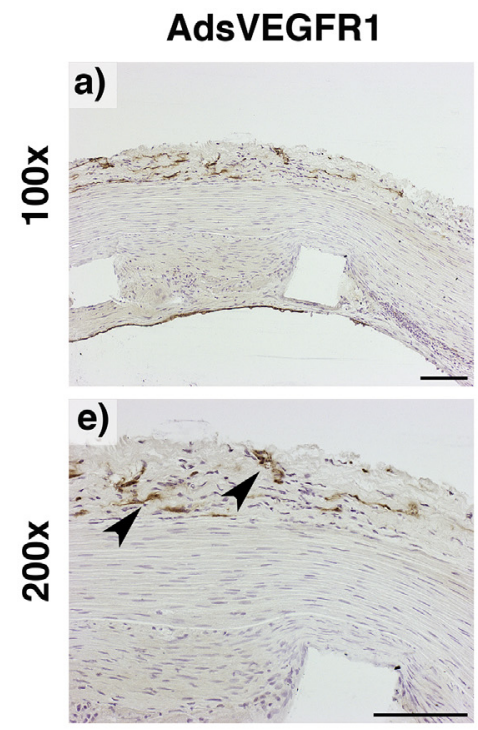

i)
AdsVEGFR2

b)
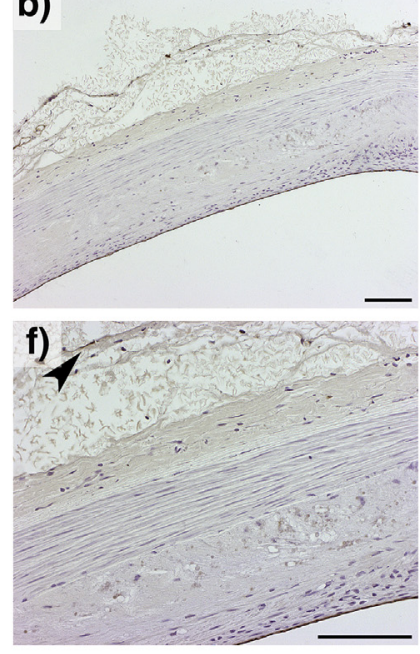

Vasa-vasorum Size

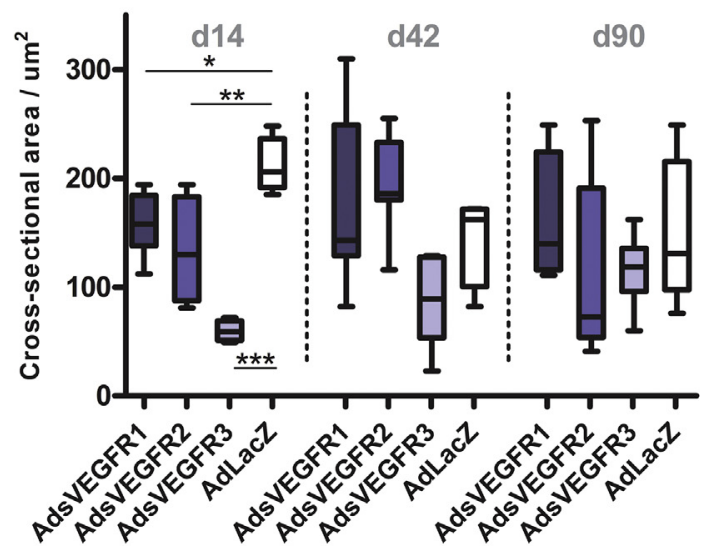

AdsVEGFR3

c)
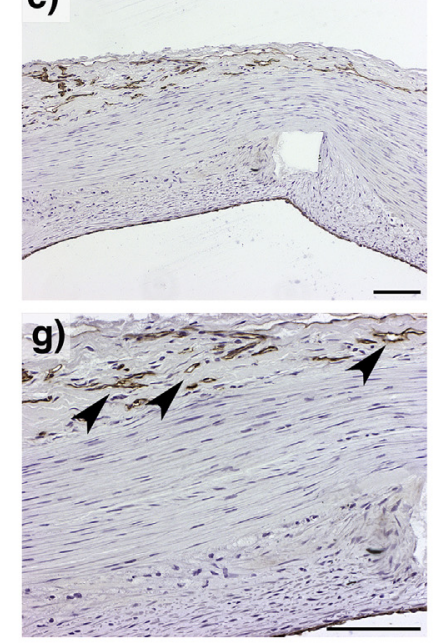

j)
AdLacZ
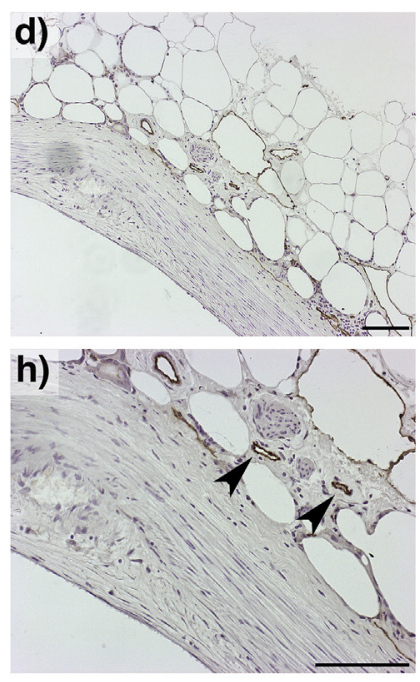

Vasa-vasorum Density

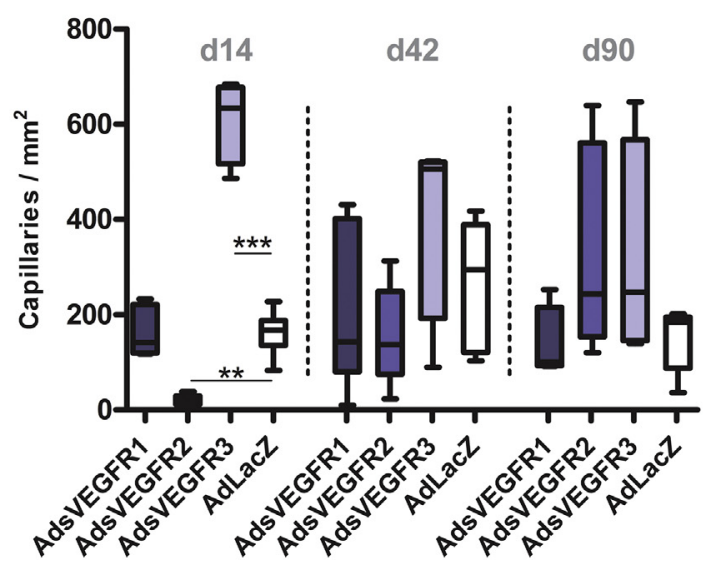

Fig. 3. Adventitial angiogenesis.

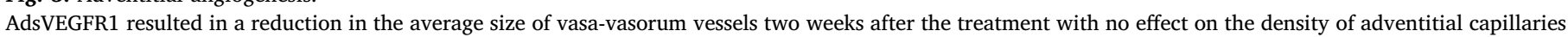

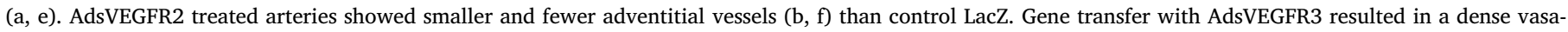

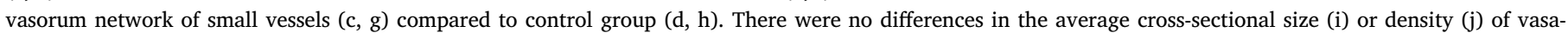

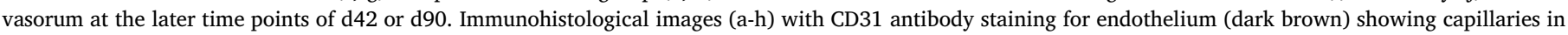

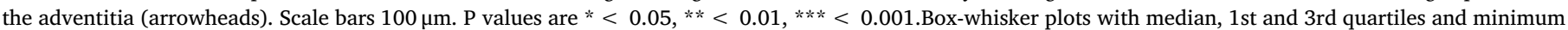

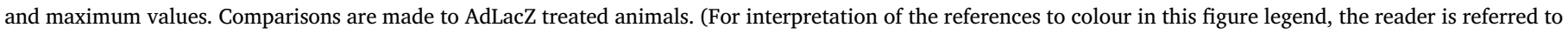
the web version of this article.)

Proliferating cell nuclei were identified in the neointima from Ki-67 staining. Data is expressed as the number of cells per $\mathrm{mm}^{2}$ of neointima. Areas identified from RAM-11 staining containing macrophages were outlined and data is shown as macrophage density in the neointima [20].

The stented aortic segments were further characterized by determining the injury score [21] and evaluating the severity and type of the atherosclerotic changes. The severity of atherosclerotic changes in neointima was assessed and a grade between I-VI [22,23] was assigned. Briefly, type I lesions show isolated foam cells, type II have fatty streaks with intracellular lipid accumulation, type III includes extracellular lipid pools, type IV lesion with a core of extracellular lipid, type $\mathrm{V}$ or fibroatheroma has a lipid core with a fibrotic layer and type VI shows characteristics of complicated lesions with possible thrombus or hemorrhagic changes. Degree of restenosis was determined from crosssectional HE stained images as ratio of neointimal area to internal elastic lamina area $\{[1-($ Luminal area/IEL area) $\times 100]\}$.

\subsection{Blood chemistry}

Blood drawn from the animals before treatment and at sacrifice were analyzed for lipid profile and signs of toxicity and included measurements for ALT, ALP, Creatinine, total cholesterol, HDL, LDL, triglycerides and urea. All analyses were done at a dedicated veterinary clinical chemistry laboratory (Movet, Kuopio, Finland).

\subsection{Statistical analysis}

All results were analyzed for statistical significance using one-way ANOVA with Dunnet's post hoc test with GraphPad Prism 6 software (GraphPad Software, La Jolla, CA, USA). P values $<0.05$ were considered significant. All data are expressed as mean \pm SD. 


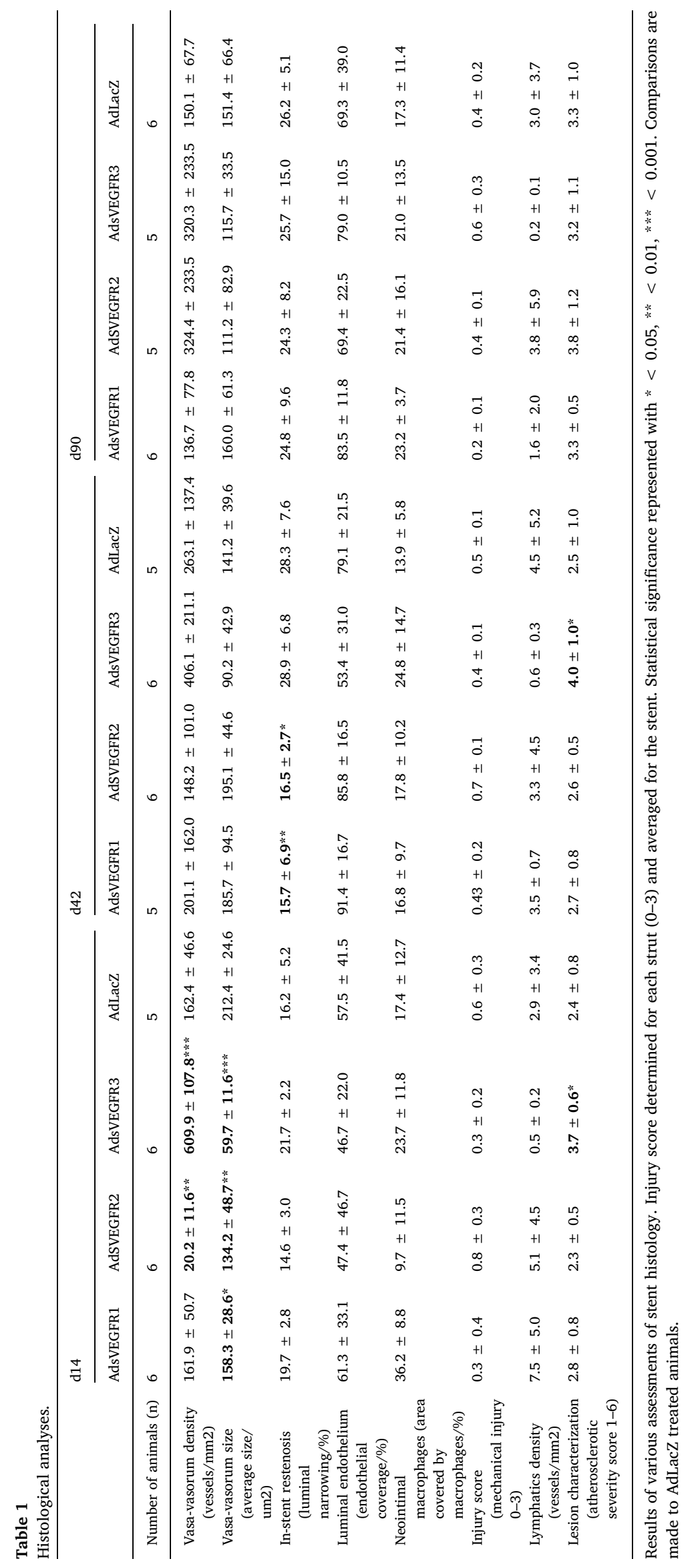


$100 x$

a)

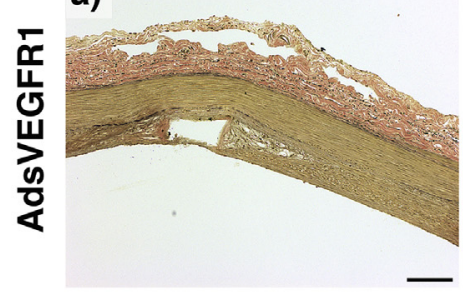

c)
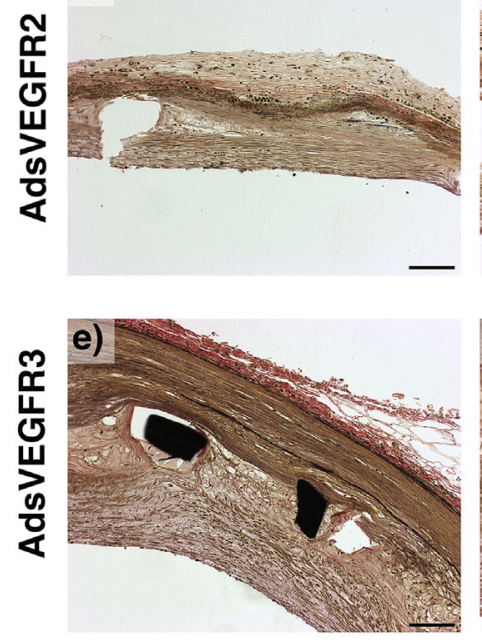

d)
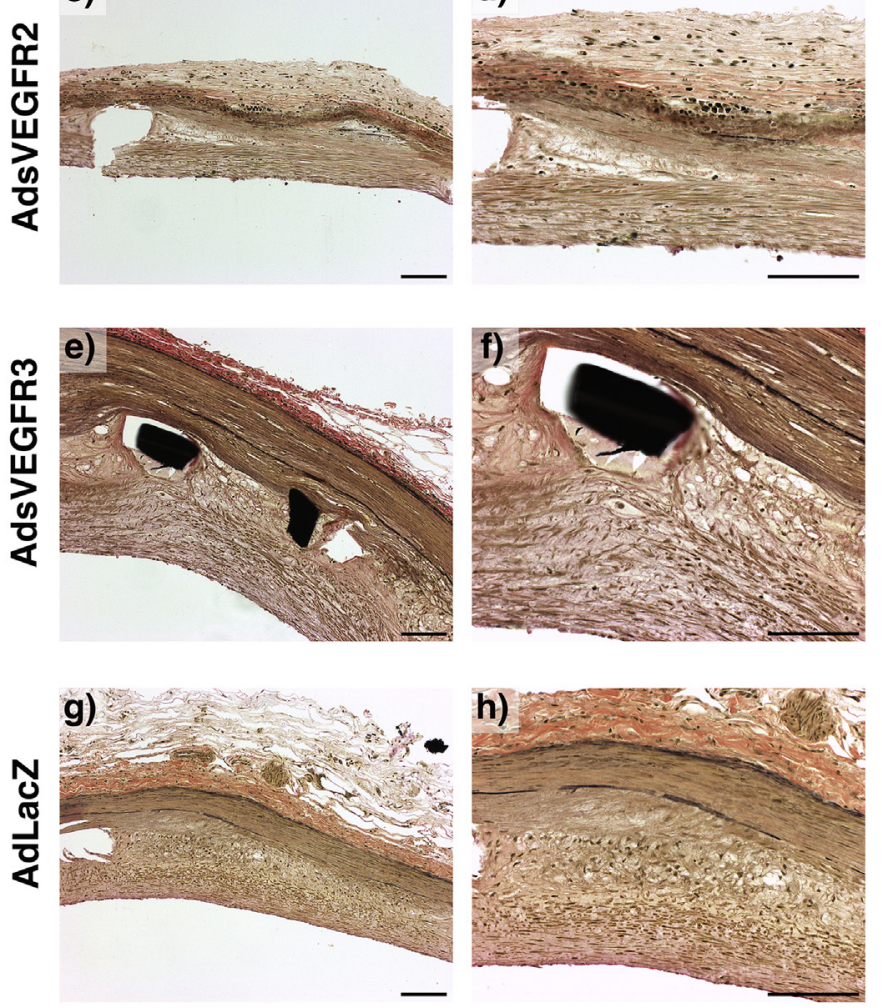

i)

\section{Restenosis}

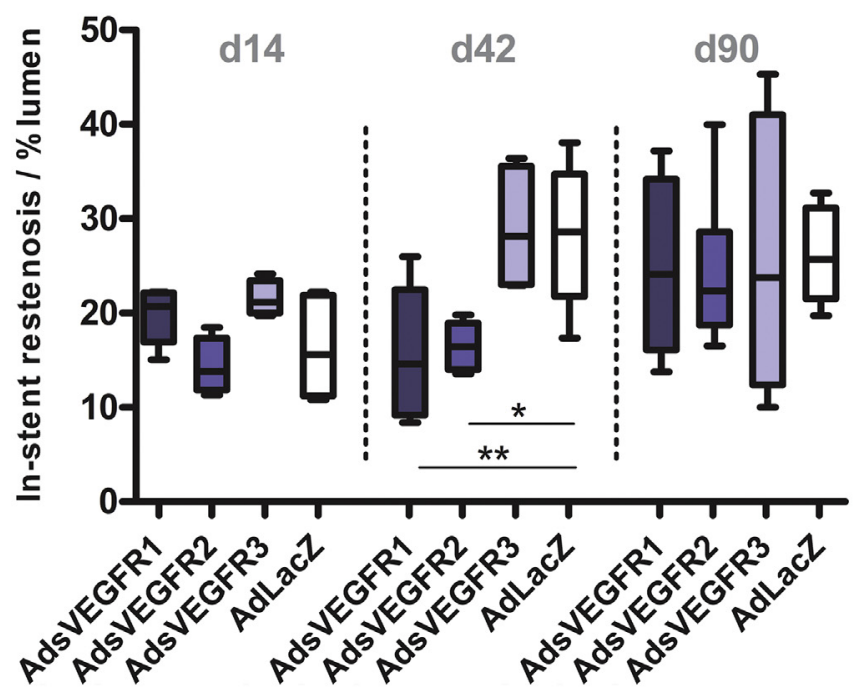

Fig. 4. In-stent restenosis.

Six weeks (d42) after gene transfer a reduction of ISR was seen with AdsVEGFR1 (a-b) and AdsVEGFR2 (c-d). AdsVEGFR3 group (e-f) did not differ from AdLacZ $(\mathrm{g}-\mathrm{h})$. No differences were seen in restenosis at the d14 or d90 time points (i). Histological stains with elastic stain $(a-h)$ where the internal elastic lamina (IEL) and external elastic lamina (EEL) are clearly visualized. Histological images on the right are higher magnification of images on the left. Scale bars $100 \mu \mathrm{m}$ in a-h. P values $*<0.05, * *<0.01$. Box-whisker plots with median, 1st and 3rd quartiles and minimum and maximum values. Comparisons are made to AdLacZ treated animals.

\section{Results}

\subsection{Gene transfer}

Marker gene expression was confirmed in LacZ treated aortas at six days after the gene transfer. $\beta$-galactosidase positive cells were detected in the connective tissue of the adventitia, mainly in fibroblasts. Positive cells were also seen in the liver surrounding the sinusoids and the spleen, but not in other collected tissue samples (Fig. 2) following AdLacZ gene transfer.

\subsection{Adventitial vasa vasorum}

Treatment with AdsVEGFR1 and AdsVEGFR2 led to a decrease in the size of adventitial vessels $14 \mathrm{~d}$ after the stenting $\left(158.3 \pm 28.6 \mu \mathrm{m}^{2}\right.$, $134.2 \pm 48.7 \mu \mathrm{m}^{2}$ and $212.4 \pm 24.6 \mu \mathrm{m}^{2}$ for AdsVEGFR1, AdsVEGFR2 and AdLacZ respectively; $\mathrm{P}<0.05$ AdsVEGFR1, $\mathrm{P}<0.01$ for AdsVEGFR2) (Fig. 3). AdsVEGFR3 treatment resulted in an even greater $\left(59.5 \mu \mathrm{m}^{2} \pm 11.6, \mathrm{P}<0.001\right)$ reduction in the average size of vasa-vasorum compared to AdLacZ. At d14, the density of capillaries was reduced in the AdsVEGFR2 treated animals whereas the treatment with AdsVEGFR3 resulted in a marked increase in the density of adventitial vasa vasorum $(20.2 \pm 11.6,609.9 \pm 107.7$ and $162.4 \pm 46.6$ vessels per $\mathrm{mm}^{2}$ for AdsVEGFR2, AdsVEGFR3 and AdLacZ, respectively; $\mathrm{P}<0.01$ for AdsVEGFR2 and $\mathrm{P}<0.001$ for AdsVEGFR3) (Table 1). There were no significant differences at later time points of 42 and 90 days (Fig. 3).

\subsection{Luminal endothelialization}

No differences were observed in the rate of luminal re-endothelialization. The endothelial layer had recovered moderately in all groups by two weeks. None of the groups showed complete endothelialization even at the three-month follow-up (Table 1).

\subsection{In-stent restenosis}

The ISR calculated as the percentage narrowing of the stented lumen was significantly decreased in the AdsVEGFR1 and AdsVEGFR2 treated groups compared to controls $42 \mathrm{~d}$ after stenting $(15.7 \pm 6.9$, $16.5 \pm 2.7$ and $28.3 \pm 7.6 \%$ restenosis for AdsVEGFR1, AdsVEGFR2 and AdLacZ, respectively; $\mathrm{P}<0.01$ for AdsVEGFR1 and $\mathrm{P}<0.05$ for AdsVEGFR2) (Fig. 4). No significant differences were observed at other time points or treatment with AdsVEGFR3 (Table 1).

\subsection{Macrophage infiltration and lesion characteristics}

Moderate macrophage infiltration was seen in all groups. The macrophage density was generally greater in areas surrounding the stent struts (Fig. 5). No differences were observed between the groups (Table 1).

The in-stent neointimal lesions displayed more advanced atherosclerotic changes at d14 in AdsVEGFR3 treated arteries compared to control with a score of $3.7 \pm 0.6$ vs. $2.4 \pm 0.8$ in AdLacZ, $\mathrm{P}<0.05$ $(2.8 \pm 0.8$ and $2.3 \pm 0.5$ for AdsVEGFR1 and AdsVEGFR2, 

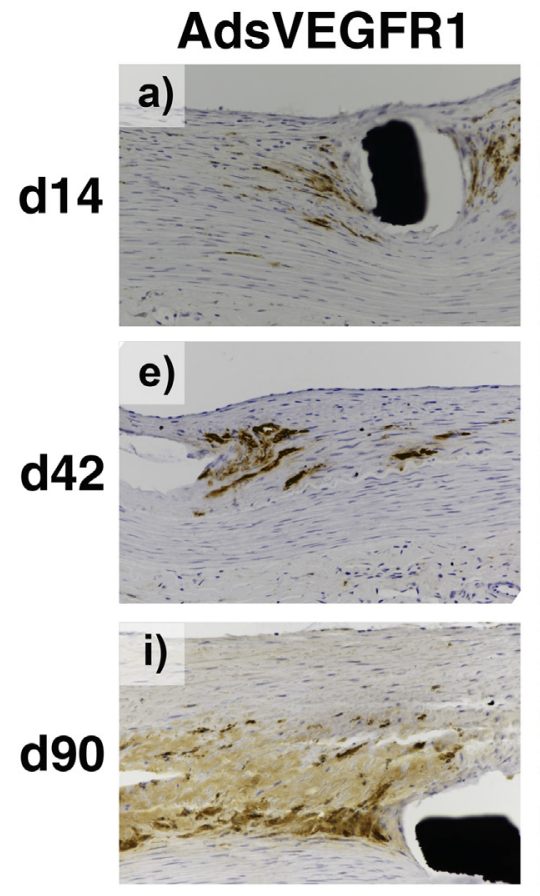

m)

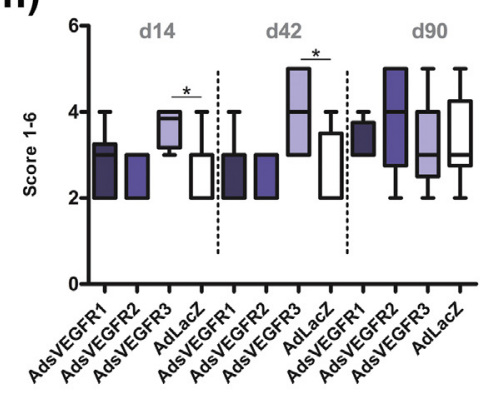

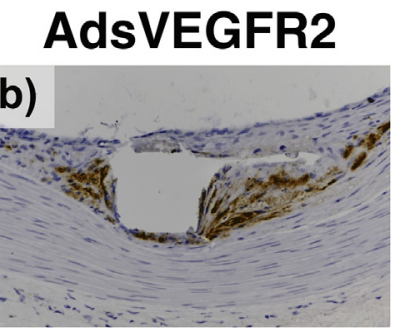

AdsVEGFR3
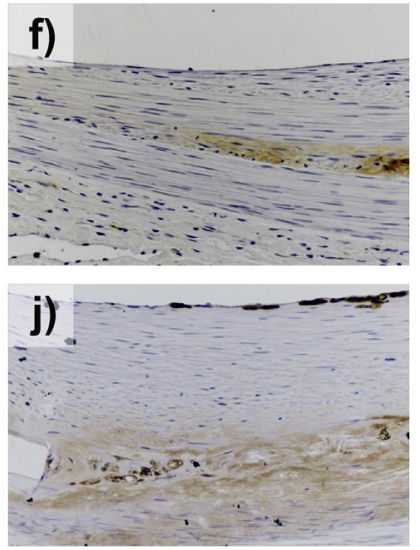

n)

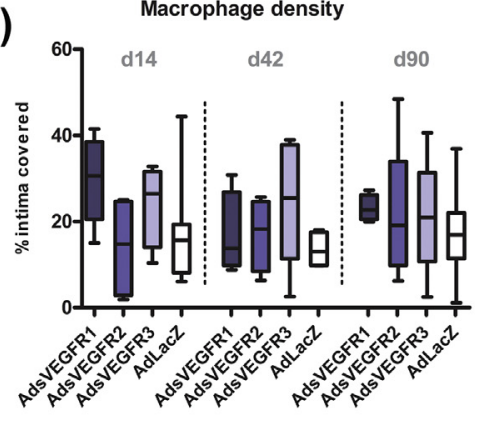

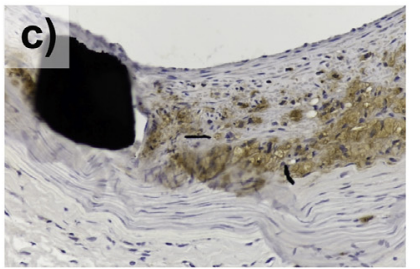
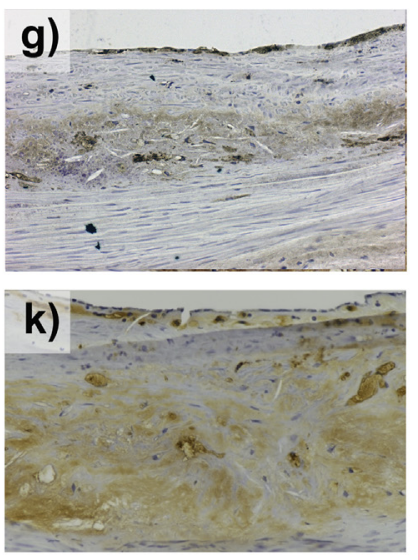

o)

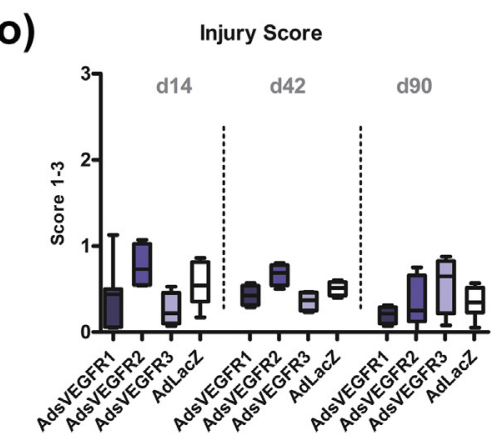

Fig. 5. Neointimal macrophages.

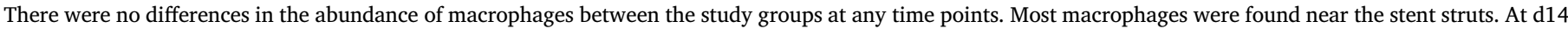

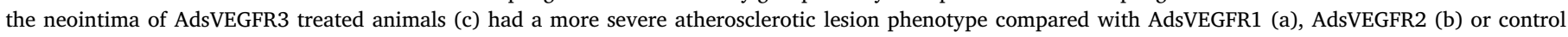

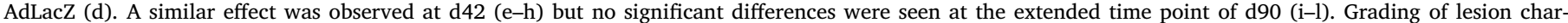

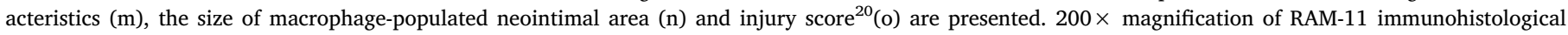

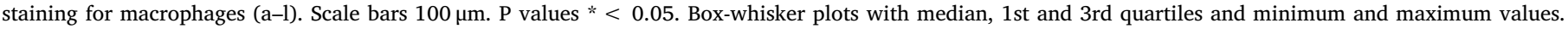
Comparisons are made to AdLacZ treated animals.

respectively, $\mathrm{P}=\mathrm{NS}$ ))(Fig. 5). The same was also seen at $\mathrm{d} 42$ with AdsVEGFR3 treated rabbits with a score of $4.0 \pm 1.0$ vs. $2.5 \pm 1.0$ in AdLacZ, $\mathrm{P}<0.05(2.7 \pm 0.8$ and $2.6 \pm 0.5$ for AdsVEGFR 1 and AdsVEGFR2, respectively, $\mathrm{P}=\mathrm{NS}$ ). There were no significant differences at $\mathrm{d} 90$.

\subsection{Adventitial lymphatic vessels}

Treatment with sVEGFR3 resulted in a trend towards reduced number of LYVE-1 positive lymphatic vessels in the adventitia at all time-points although the difference was not statistically significant (Fig. 6). Gene transfers with other sVEGFRs did not influence the growth of lymphatic vessels (Table 1).

\subsection{Neointimal proliferation}

No statistically significant differences were observed in the number of proliferating cells in the neointima in any of the time points. At d14 proliferation counts were $62.2 \pm 27.2,58.2 \pm 15.1,58,1 \pm 23.4$ and $43.3 \pm 23.8$ per square $\mathrm{mm}$ of neointima for AdLacZ, AdsVEGFR1,
AdsVEGFR2 and AdsVEGFR3, respectively. Number of proliferating cells was reduced in all groups at d42 with $4.6 \pm 1.5,4.1 \pm 4.6$, $6.1 \pm 3.0$ and $3.5 \pm 3.3$ for AdLacZ, AdsVEGFR1, AdsVEGFR2 and AdsVEGFR3, respectively, $\mathrm{P}=\mathrm{NS}$. Proliferation rates at $\mathrm{d} 90$ were $11.5 \pm 5.6,14.5 \pm 6.5,9.9 \pm 3.7$ and $10.2 \pm 4.3$ for AdLacZ, AdsVEGFR1, AdsVEGFR2 and AdsVEGFR3, respectively, P = NS.

\subsection{Blood chemistry and safety analyses}

No signs of toxicity or side effects were found based on clinical chemistry (Table 2) and histopathological analysis of the major organs (Supplementary Figs. 1-5). There were no differences between the groups in local inflammatory responses, neointimal proliferation or injury scores (Table 1).

\section{Discussion}

The role of vasa vasorum in the formation of primary atherosclerotic lesions has previously been suggested [11]. In this study we show that by blocking angiogenic signaling using adventitial expression of decoy 


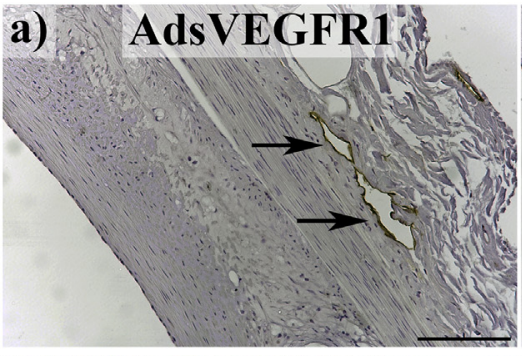

b) AdsVEGFR2

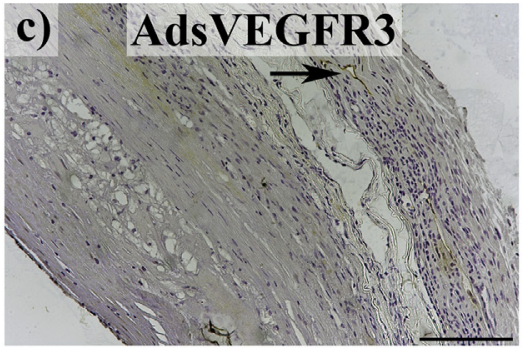

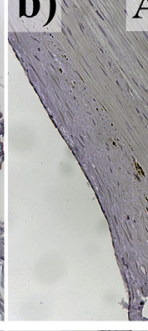

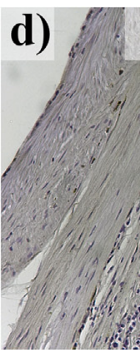

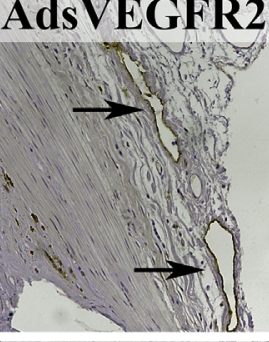

AdLacZ

Fig. 6dventitial lymphatics.

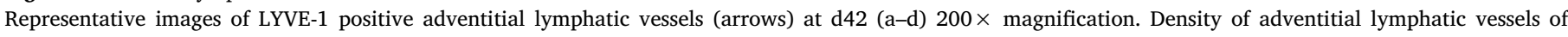

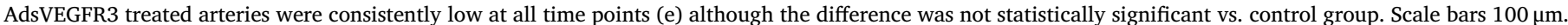
Box-whisker plots with median, 1st and 3rd quartiles and minimum and maximum values. Comparisons are made to AdLacZ treated animals.

receptors for VEGFR1 or VEGFR2 reduces the number and size of adventitial vasa vasorum, and leads to reduction of ISR. Conversely, periadventitial targeting of pro lymphangiogenic VEGFR3 activation with decoy inhibitors resulted in a more adverse in-stent neointimal atherosclerotic phenotype. We utilized an atherosclerotic model in which a lesion is created in a hypercholesterolemic animal with additional balloon injury prior to the stenting procedure to mimic the clinical setting of coronary stenting. Time-points were selected to address the acute changes after peak transgene expression at $\mathrm{d} 14$, the subacute changes in restenosis formation at $\mathrm{d} 42$ and chronic lesion development at $\mathrm{d} 90$. Use of the intermediate $\mathrm{d} 42$ time-point is well documented in prior literature [24,25]. Limitations of the study are the lack of a solvent group with injections lacking viral particles and a stent group without drug delivery catheter intervention.

Treatment with AdsVEGFR1 and AdsVEGFR2 resulted in an attenuated adventitial angiogenic response two weeks after the treatment, following the peak transgene expression of adenovirus vector system [26]. Possibly due to the limited duration of expression of adenoviruses, the effect on the vasa vasorum size and density was lost at the later time points. The main angiogenic signaling occurs via the VEGFR2 pathway whereas the VEGFR1 acts partly as a decoy receptor in the physiological setting [12]. In this study, treatment with AdsVEGFR2 resulted in both fewer and smaller adventitial vessels while AdsVEGFR1 treatment only affected the size of vasa-vasorum suggesting that targeting VEGFR2 pathway might be the most potent approach for the inhibition of the growth of vasa vasorum. However, both AdsVEGFR1 and AdsVEGFR2 treatments resulted in similar reductions in ISR at six weeks follow up.

Following the impaired adventitial angiogenesis at two weeks, both the AdsVEGFR1 and AdsVEGFR2 treated animals showed a reduction in ISR six weeks after the treatment. The reduced angiogenesis seen at the two-week time point likely contributes to the reduced ISR four weeks later. In fact it has been shown that the adventitial tissue surrounding arterial stents is hypoxic in nature and this hypoxia leads to the growth of adventitial vessels, this adventitial angiogenesis is reduced by AdsVEGFR1 and AdsVEGFR2 therapy in this study [27]. Interestingly, a similar degree of reduction in ISR was seen with AdsVEGFR1 although its effects on vasa-vasorum at $\mathrm{d} 14$ were more limited compared to AdsVEGFR2 therapy. The results are in line with previous findings showing increased neointima formation in rabbit carotid arteries following adventitial VEGF gene transfers [28]. Similar to the loss of the anti-angiogenic effect, no effects on ISR were observed at the extended follow-up of 90 days. The transient therapeutic effects suggests that an alternative vector system or other delivery method should be considered in the future to accomplish more sustained reduction in ISR.

The in-stent neointima formation observed already at the two-week time-point represents more than half of the ISR seen at the extended follow-up of $90 \mathrm{~d}$ in all treatment groups. This finding confirms that the acute phase of 1-2 weeks after the stent placement is critical for the healing process of the artery and the onset of ISR [29]. While a drug eluting stent will potentially start the release of therapeutic agent immediately after stent implantation, all gene therapy based approaches have the limitation of delayed onset of therapy as the therapeutic protein will only be produced after sufficient transduction of the transgene.

The rate of regeneration of the luminal endothelium was similar in all groups at all time points and was not influenced by the transient adventitial expression of anti-angiogenic decoy receptors. Physiological VEGF signaling maintains endothelial homeostasis [30] and is necessary for endothelial recovery after stenting. The adventitial expression in this study seems to limit therapeutic effects of the soluble receptor proteins mainly to the adventitia and enables paracrine actions of the decoy inhibitors thus allowing the luminal endothelium to regenerate to the same extent in the treated and control arteries. The lack of complete re-endothelialization even at d90 confirms the clinical relevance of the WHHL rabbit model used in this study mimicking severe atherosclerosis and chronic endothelial dysfunction present in patients with occlusive arterial disease. No signs of toxicity or adverse effects were observed.

Treatment with AdsVEGFR3 also seemed to alter angiogenesis in the stented artery. At two weeks AdsVEGFR3 treated animals displayed a marked increase in the capillary density around stented vessel segments while the average size of the new vessels remained notably small. This effect on angiogenesis did not alter ISR at the intermediate time point of d42 unlike the treatments with AdsVEGFR1 and AdsVEGFR2. Reduction of VEGFR3 signaling has been shown to interfere with Notch signaling resulting in a hypervascular phenotype of angiogenesis with increased sprouting [31]. It is possible that AdsVEGFR3 administered here has had a similar effect on the adventitial vasculature. Treatment with AdsVEGFR3 also resulted in a statistically non-significant trend towards blockage of lymphangiogenesis at all time points.

Treatment with AdsVEGFR3 led to an altered composition of the developing neointima, which was observed already at d14 (Fig. 5). 
AdsVEGFR3 treated arteries showed more complicated neointimal changes with higher atherosclerotic lesion scores [20,21]. Increased VEGFR3 signaling with periluminal delivery of AdVEGF-C has in the past been shown to limit intimal thickening following balloon angioplasty in a rabbit model [32]. Here we show more complicated neointimal lesions with an intervention that reduces periadventitial VEGFR3 activation following stent placement in atherosclerotic arteries. Local lymphatics have been suggested to contribute to the drainage of interstitial fluid and cholesterol from the atherosclerotic arterial wall $[14,33]$. Taken together, local activation of VEGFR3 may present a new possibility to treat restenotic arterial disease and promote healing after stent implantation.

\section{Conclusions}

This study suggests that adventitial plaque angiogenesis plays an important role in the pathogenesis of ISR. Importantly, the abluminal delivery of anti-angiogenic agents reduced ISR but did not affect the regeneration of luminal endothelium. Conversely, periadventitial inhibition of lymphangiogenic signaling adversely influenced healing after stent implantation. Therefore, local gene therapy is a potential new way to reduce adventitial angiogenesis and ISR in patients who require angioplasty and stent placement.

Supplementary data to this article can be found online at https:// doi.org/10.1016/j.yjmcc.2018.07.007.

\section{Disclosures}

None.

\section{Acknowledgements}

This study was supported by the Academy of Finland307402 Centres of Excellence, Finnish Foundation for Cardiovascular Diseases, Sigrid Juselius foundation, Aarne Koskelo foundation, The Finnish Medical Foundation, Päivikki and Sakari Sohlberg Foundation and the Finnish Cultural Foundation.

\section{References}

[1] A.J. Lusis, Atherosclerosis, Nature 407 (6801) (September 14, 2000) 233-241.

[2] D.L. Fischman, M.B. Leon, D.S. Baim, R.A. Schatz, M.P. Savage, I. Penn, et al., for the Stent Restenosis Study Investigators, a randomized comparison of coronarystent placement and balloon angioplasty in the treatment of coronary artery disease. Stent Restenosis Study Investigators, N. Engl. J. Med. 331 (8) (August 25, 1994) 496-501.

[3] U. Sigwart, J. Puel, V. Mirkovitch, F. Joffre, L. Kappenberger, Intravascular stents to prevent occlusion and restenosis after transluminal angioplasty, N. Engl. J. Med. 316 (12) (March 19, 1987) 701-706.

[4] R. Hoffmann, G.S. Mintz, G.R. Dussaillant, J.J. Popma, A.D. Pichard, L.F. Satler, et al., Patterns and mechanisms of in-stent restenosis. A serial intravascular ultrasound study, Circulation 94 (6) (September 15, 1996) 1247-1254.

[5] C. Stettler, S. Wandel, S. Allemann, A. Kastrati, M.C. Morice, A. Schömig, et al., Outcomes associated with drug-eluting and bare-metal stents: a collaborative network meta-analysis, Lancet 370 (9591) (September 15, 2007) 937-948.

[6] Inoue T1, K. Croce, T. Morooka, M. Sakuma, K. Node, D.I. Simon, Vascular inflammation and repair: implications for re-endothelialization, restenosis, and stent thrombosis, JACC Cardiovasc. Interv. 4 (10) (October, 2011) 1057-1066.

[7] S. Ylä-Herttuala, J.F. Bentzon, M. Daemen, E. Falk, H.M. Garcia-Garcia, J. Herrmann, et al., ESC Working Group of Atherosclerosis and Vascular Biology. Stabilization of atherosclerotic plaques: an update, Eur. Heart J. 34 (42) (November, 2013) 3251-3258.

[8] O. Leppänen, T. Björnheden, M. Evaldsson, J. Borén, O. Wiklund, M. Levin, ATP depletion in macrophages in the core of advanced rabbit atherosclerotic plaques in vivo, Atherosclerosis 188 (2) (October, 2006) 323-330.

[9] M. Levin, O. Leppänen, M. Evaldsson, O. Wiklund, G. Bondjers, T. Björnheden, Mapping of ATP, glucose, glycogen, and lactate concentrations within the arterial wall, Arterioscler. Thromb. Vasc. Biol. 23 (10) (October 1, 2003) 1801-1807.

[10] J.C. Sluimer, J.M. Gasc, J.L. van Wanroij, N. Kisters, M. Groeneweg, M.D. Sollewijn Gelpke, et al., Hypoxia, hypoxia-inducible transcription factor, and macrophages in human atherosclerotic plaques are correlated with intraplaque angiogenesis, J. Am Coll. Cardiol. 51 (13) (April 1, 2008) 1258-1265.

[11] R. Virmani, F.D. Kolodgie, A.P. Burke, A.V. Finn, H.K. Gold, T.N. Tulenko, et al., 
Atherosclerotic plaque progression and vulnerability to rupture: angiogenesis as a source of intraplaque hemorrhage, Arterioscler. Thromb. Vasc. Biol. 25 (10) (October, 2005) 2054-2061.

[12] M. Shibuya, L. Claesson-Welsh, Signal transduction by VEGF receptors in regulation of angiogenesis and lymphangiogenesis, Exp. Cell Res. 312 (5) (March 10, 2006) 549-560.

[13] H.M. Kwon, G. Sangiorgi, E.L. Ritman, A. Lerman, C. McKenna, R. Virmani, et al., Adventitial vasa vasorum in balloon-injured coronary arteries: visualization and quantitation by a microscopic three-dimensional computed tomography technique, J. Am. Coll. Cardiol. 32 (7) (December, 1998) 2072-2079.

[14] T. Vuorio, H. Nurmi, K. Moulton, J. Kurkipuro, M.R. Robciuc, M. Ohman, et al., Lymphatic vessel insufficiency in hypercholesterolemic mice alters lipoprotein levels and promotes atherogenesis, Arterioscler. Thromb. Vasc. Biol. 34 (6) (June, 2014) 1162-1170.

[15] J.H. Bräsen, O. Leppänen, M. Inkala, T. Heikura, M. Levin, F. Ahrens, et al., Extracellular superoxide dismutase accelerates endothelial recovery and inhibits instent restenosis in stented atherosclerotic Watanabe heritable hyperlipidemic rabbit aorta, J. Am. Coll. Cardiol. 50 (23) (December 4, 2007) 2249-2253.

[16] C.M. Failla, M. Carbo, V. Morea, Positive and negative regulation of angiogenesis by soluble vascular endothelial growth factor Receptor-1, Int. J. Mol. Sci. 19 (5) (April 27, 2018).

[17] M. Hedman, J. Hartikainen, M. Syvänne, J. Stjernvall, A. Hedman, A. Kivelä, et al., Safety and feasibility of catheter-based local intracoronary vascular endothelial growth factor gene transfer in the prevention of postangioplasty and in-stent restenosis and in the treatment of chronic myocardial ischemia: phase II results of the Kuopio Angiogenesis Trial (KAT), Circulation 107 (21) (June 3, 2003) 2677-2683.

[18] M. Sopo, M. Anttila, H. Sallinen, L. Tuppurainen, A. Laurema, S. Laidinen, et al., Antiangiogenic gene therapy with soluble VEGF-receptors $-1,-2$ and -3 together with paclitaxel prolongs survival of mice with human ovarian carcinoma, Int. J. Cancer 131 (10) (November 15, 2012) 2394-2401, https://doi.org/10.1002/ijc. 27495 (Epub 2012 Mar 27).

[19] P. Korpisalo, J.P. Hytönen, J.T. Laitinen, S. Laidinen, H. Parviainen, H. Karvinen, et al., Capillary enlargement, not sprouting angiogenesis, determines beneficial therapeutic effects and side effects of angiogenic gene therapy, Eur. Heart J. 32 (13) (July, 2011) 1664-1672.

[20] O. Leppänen, J. Rutanen, M.O. Hiltunen, T.T. Rissanen, M.P. Turunen, T. Sjöblom, et al., Oral imatinib mesylate (STI571/gleevec) improves the efficacy of local intravascular vascular endothelial growth factor-C gene transfer in reducing neointimal growth in hypercholesterolemic rabbits, Circulation 109 (9) (March 9, 2004) 1140-1146.

[21] R.S. Schwartz, K.C. Huber, J.G. Murphy, W.D. Edwards, A.R. Camrud, R.E. Vlietstra, et al., Restenosis and the proportional neointimal response to coronary artery injury: results in a porcine model, J. Am. Coll. Cardiol. 19 (2) (February, 1992) 267-274.

[22] H.C. Stary, A.B. Chandler, S. Glagov, J.R. Guyton, W. Insull Jr., M.E. Rosenfeld, et al., A definition of initial, fatty streak, and intermediate lesions of atherosclerosis. A report from the Committee on Vascular Lesions of the Council on Arteriosclerosis, American Heart Association, Arterioscler. Thromb. 14 (5) (May, 1994) 840-856.

[23] H.C. Stary, A.B. Chandler, R.E. Dinsmore, V. Fuster, S. Glagov, W. Insull Jr.et al., A definition of advanced types of atherosclerotic lesions and a histological classification of atherosclerosis. A report from the committee on vascular lesions of the council on arteriosclerosis, American Heart Association, Circulation 92 (5) (September 1, 1995) 1355-1374.

[24] F. Ribichini, M. Joner, V. Ferrero, A.V. Finn, J. Crimins, G. Nakazawa, et al., Effects of oral prednisone after stenting in a rabbit model of established atherosclerosis, J. Am. Coll. Cardiol. 50 (2) (July 10, 2007) 176-185 (Epub 2007 Jun 22).

[25] M. Joner, K. Morimoto, H. Kasukawa, K. Steigerwald, S. Merl, G. Nakazawa, et al., Site-specific targeting of nanoparticle prednisolone reduces in-stent restenosis in a rabbit model of established atheroma, Arterioscler. Thromb. Vasc. Biol. 28 (11) (November, 2008) 1960-1966.

[26] S. Ylä-Herttuala, C. Bridges, M.G. Katz, P. Korpisalo, Angiogenic gene therapy in cardiovascular diseases: dream or vision? Eur. Heart J. 38 (18) (May 7, 2017) 1365-1371.

[27] S. Bhardwaj, H. Roy, M. Gruchala, H. Viita, I. Kholova, I. Kokina, et al., Angiogenic responses of vascular endothelial growth factors in periadventitial tissue, Hum. Gene Ther. 14 (15) (October 10, 2003) 1451-1462.

[28] S. Bhardwaj, H. Roy, T. Heikura, S. Ylä-Herttuala, VEGF-A, VEGF-D and VEGF-D (DeltaNDeltaC) induced intimal hyperplasia in carotid arteries, Eur. J. Clin. Investig. 35 (11) (November, 2005) 669-676.

[29] M.A. Costa, D.I. Simon, Molecular basis of restenosis and drug-eluting stents, Circulation 111 (17) (May 3, 2005) 2257-2273.

[30] S. Lee, T.T. Chen, C.L. Barber, M.C. Jordan, J. Murdock, S. Desai, et al., Autocrine VEGF signaling is required for vascular homeostasis, Cell 130 (4) (August 24, 2007) $691-703$

[31] T. Tammela, G. Zarkada, H. Nurmi, L. Jakobsson, K. Heinolainen, D. Tvorogov, et al., VEGFR-3 controls tip to stalk conversion at vessel fusion sites by reinforcing Notch signalling, Nat. Cell Biol. 13 (10) (Sep 11, 2011) 1202-1213.

[32] M.O. Hiltunen, M. Laitinen, M.P. Turunen, M. Jeltsch, J. Hartikainen, T.T. Rissanen, et al., Intravascular adenovirus-mediated VEGF-C gene transfer reduces neointima formation in balloon-denuded rabbit aorta, Circulation 102 (18) (October 31, 2000) 2262-2268.

[33] C. Martel, W. Li, B. Fulp, A.M. Platt, E.L. Gautier, M. Westerterp, et al., Lymphatic vasculature mediates macrophage reverse cholesterol transport in mice, J. Clin. Invest. 123 (4) (April, 2013) 1571-1579. 